\title{
УДК: 519.95
}

Название: Некоторые модели анализа и прогнозирования временных рядов

\section{Автор(ы):}

Шевченко И.В. (Институт систем информатики им А.П. Ершова СО РАН),

Аннотация: В статье рассматриваются несколько популярных классических моделей анализа и прогнозирования временных рядов. Вначале описываются относительно простые модели усреднения и сглаживания, затем модели авторегрессии, скользящего среднего, а также «смешанная» модель авторегрессии-скользящего среднего, полученная путем скрещивания двух последних моделей. Последней рассматривается интегрированная модель авторегрессии-скользящего среднего для случая нестационарных временных рядов.

Ключевые слова: прогнозирование, временной ряд, усреднение, экспоненциальное сглаживание, модель авторегрессии, скользящее среднее

1. Введение. Задача прогнозирования неопределенного будущего всегда была актуальна во многих областях. В данном случае, говоря о прогнозировании, мы подразумеваем анализ и прогнозирование временных рядов - наборов данных, которые были собраны или зафиксированы через последовательные промежутки времени. Существует огромное множество методов для выполнения этой задачи - начиная от простого экспоненциального сглаживания и заканчивая нейронными сетями. Многие методики стали неотъемлемой частью таких областей, как, например, эконометрика. Суть этих методов, если говорить в общем, состоит в подборе моделей, эффективно описывающих данные и способных быть продолженными в будущее.

Необходимость в методах прогнозирования обуславливается тем, что человек, обладая поразительными аналитическими способностями, а также знаниями и интуицией, склонен привносить в свои прогнозы некоторую степень субъективизма и недооценивать те или иные факторы [8].

Ниже рассматриваются наиболее популярные классические методы прогнозирования. Наиболее значимыми характеристиками при выборе той или иной модели прогнозирования являются, прежде всего, модель данных, на которые она ориентирована, и временная отдаленность выдаваемых ею прогнозов. Поэтому выбору модели предшествует анализ общей структуры ряда - чему и посвящен материал, предшествующий описаниям конкретных моделей прогнозирования.

2. Автокорреляция и частная автокорреляция. Главным отличием временного ряда от случайной последовательности является тот факт, что его члены являются взаимозависимыми. Степень связи значений двух случайных величин может быть выражена 
коэффициентом корреляции между ними. Соответственно, когда мы хотим исследовать ряд на связь между его последовательными членами, разнесенными во времени на один и более периодов, мы аналогично можем вычислить коэффициент корреляции. В данном случае логичнее называть такие коэффициенты - автокорреляциями [4].

Иными словами автокоррелящуия - корреляция между величиной и ее запаздыванием в один и более периодов времени. Число периодов, по которым рассчитывается коэффициент автокорреляции, часто называют лагом или порядком автокорреляции.

Следующая формула показывает, как вычисляется коэффициент автокорреляции $r_{k}$ между наблюдениями $Y_{\mathrm{t}}$ и $Y_{\mathrm{t}-1}$, - т.е. с запаздыванием на k периодов:

$$
r_{k}=\frac{\sum_{t=k+1}^{n}\left(Y_{t}-\bar{Y}\right)\left(Y_{t-k}-\bar{Y}\right)}{\sum_{t=1}^{n}\left(Y_{t}-\bar{Y}\right)^{2}}
$$

где

$r_{k}-$ коэффициент автокорреляции с запаздыванием на k периодов;

$\bar{Y}$ - среднее значение ряда;

$Y_{t}$ - наблюдение (отклик) в момент времени t;

Частная автокоррелящ̧ия за промежуток времени $\mathrm{k}$ - это корреляция между $Y_{t}$ и $Y_{t-k}$, т.е. отклик для периодов $\mathrm{t}$ и t-k после устранения влияния промежуточных значений $Y_{t-1}, Y_{t-2}$, $\ldots, Y_{t-k+1}$

Коррелограммой, или автокорреляционной функциией, является график коэффициентов автокорреляции для различных запаздываний во времени для заданного временного ряда. Анализ автокорреляционной функции и коррелограммы позволяет определить лаг, при котором автокорреляция наиболее высокая, а, следовательно, и лаг, при котором связь между текущим и предыдущими уровнями ряда наиболее тесная, т.е. с помощью анализа автокорреляционной функции и коррелограммы можно выявить структуру ряда.

Использование коэффициентов автокорреляции помогает в изучении временного ряда, давая ответ на основные интересующие нас вопросы: являются ли данные случайными, является ли ряд стационарным, или напротив, имеются ли в нем сезонные колебания?

И так, при анализе структуры временного ряда первый разумный вопрос, который должен возникать - есть ли в данных вообще какие-либо зависимости, является ли он случайным? Иными словами, нам необходимо выяснить есть ли зависимость между последовательными членами ряда. Анализ автокорреляций в данном случае может нам помочь. Если временной ряд имеет случайную природу, то коэффициенты автокорреляции для любого лага будут близки к нулю. 
Следующий вопрос - имеют ли данные тренд? Тренд - долгосрочная компонента, отражающая возрастание или убывание временного ряда в течении длительного периода времени [7]. На рис. 1 можно наблюдать пример трендовой последовательности. Временной ряд, имеющий тренд, также называют нестационарныл. Если ряд имеет тренд, то существует заметная тенденция в последовательности его членов. Соответственно, автокорреляции такого ряда будут иметь убывающий к нулю вид. Зачастую для анализа нестационарных рядов из них различными способами предварительно удаляется трендовая составляющая. Для этого, например, можно прибегнуть к взятию разности ряда, т.е. вместо исходного ряда

$$
Y_{0}, Y_{1}, Y_{2}, Y_{3, \ldots}
$$

рассматривать ряд вида:

$$
Y_{1}-Y_{0}, Y_{2}-Y_{1}, Y_{3}-Y_{2} \ldots
$$

Временные ряды, имеющие трендовую компоненту, также могут иметь циклическую компоненту - волнообразные флуктуации вокруг тренда.

Стационарным рядом называется ряд, основные статистические характеристики которого, такие как среднее значение и дисперсия, остаются постоянными во времени [7]. Таким образом, стационарный ряд колеблется вокруг некоторого фиксированного уровня или в канале (рис. 2). Можно заметить, что ряд, имеющий тренд, не является стационарным. Коэффициенты автокорреляции стационарного ряда убывают достаточно быстро.

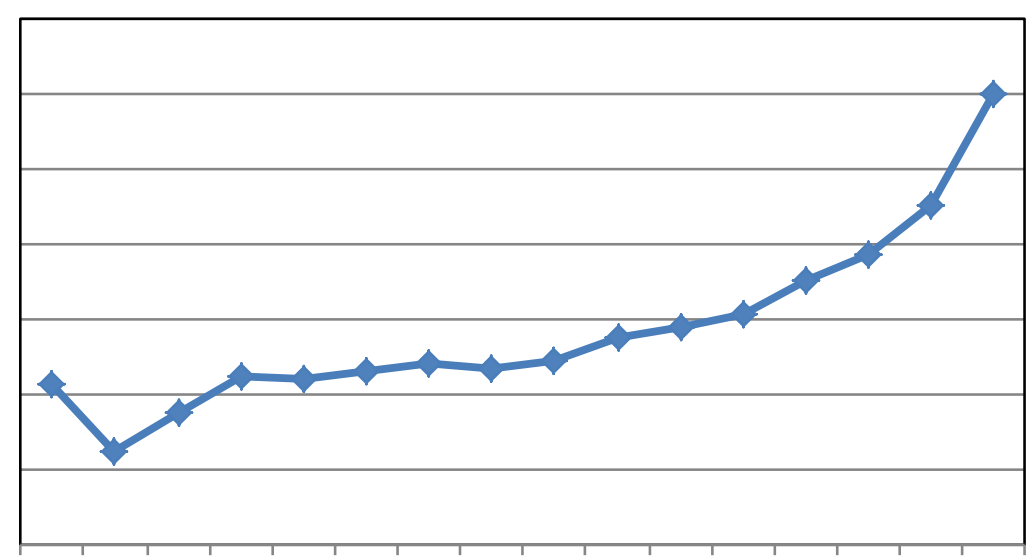

Рис. 1. Рид с трендовой составляющей 
Если данные имеют некоторый периодичный, повторяющийся характер, то говорят, что в них проявляется сезонная компонента. Таким образом, сезонной компонентой называют периодические изменения в данных, повторяющиеся, например, из года в год. Это должно отражаться в виде значительных коэффициентов автокорреляции. Например, если ряд имеет годовую сезонность, и, скажем, соответствующие месяцы каждого года очень похожи, значит, стоит ожидать больших значений для автокорреляций с запаздыванием в 12 периодов.

3. Измерение ошибки прогноза. Ошибка прогноза есть мера отклонения прогноза от реального значения некоторого члена ряда. Если обозначить прогноз значения ряда $Y_{t}$ в

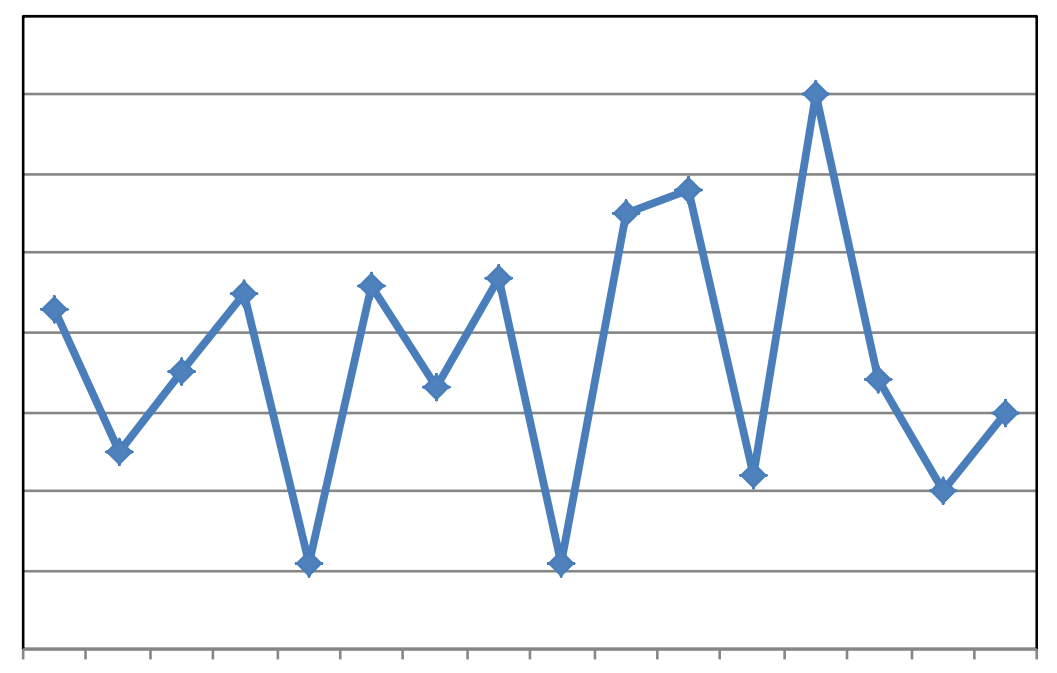

Рис. 2. Стационарный ряд

момент времени $t$ за $\widehat{Y}_{t}$, то нас будет интересовать обычно средняя оценка расхождений $\widehat{Y}_{t}$ от величины $Y_{t}$. При этом естественным будет брать значение ошибки в каждый конкретный момент времени за $Y_{t}-\widehat{Y}$.

Существуют разные методики оценки прогноза. Например, простое усреднение абсолютной величины отклонения:

$$
E=\frac{1}{n} \sum_{t=1}^{n}\left|Y_{t}-\hat{Y}\right|
$$

где $E$ - ошибка прогноза. Суть данной оценки состоит в том, чтобы измерить среднюю величину отклонения в тех же величинах, что и исходные значения ряда.

Если же абсолютные значения ряда нам не так важны или мало о чем говорят, бывает полезно смотреть на относительное отклонение прогноза, т.е. отклонения относительно 
абсолютных значений ряда. Для этого необходимо просто разделить предыдущую оценку на значение ряда в момент времени $t$ :

$$
E=\frac{1}{n} \sum_{t=1}^{n} \frac{\left|Y_{t}-\hat{Y}\right|}{Y_{t}}
$$

можно также ошибку прогноза для каждого наблюдения определять в процентах по модулю:

$$
E=\frac{1}{n} \sum_{t=1}^{n} \frac{\left|Y_{t}-\hat{Y}\right|}{Y_{t}} \times 100
$$

Следующий довольно распространенный способ оценки прогноза - среднеквадратичная ошибка.

$$
E=\frac{1}{n} \sum_{t=1}^{n}\left(Y_{t}-\hat{Y}\right)^{2}
$$

Особенность ее заключается в том, что методы, дающие более стабильную (равномерную) ошибку прогноза, будут иметь лучшую оценку, чем те, что имеют редкие, но значительные отклонения в прогнозе.

Существует множество аналогичных способов измерять ошибку прогноза. Выбор конкретной оценки зависит от конкретных задач.

4. Наивные модели. По названию можно догадаться, что это один из самых простых классов моделей. Суть методов заключается в предположении, что будущее лучше всего описывается самыми свежими данными [7]. Самый простой пример - предсказание типа «завтра будет так же, как сегодня», если обозначить прогнозируемую величину за $\widetilde{Y}_{t+1}$ :

$$
\widehat{Y}_{t+1}=Y_{t}
$$

Естественно предположить, что проблема данной модели в том, что случайные флуктуации сильно портят прогноз. Но, тем не менее, в условиях, например, недостатка исторических данных для анализа трудно придумать иную альтернативу.

Улучшить прогноз в данном случае может помочь изучение структуры ряда. Если, например, есть предположение, что в данных имеется трендовая составляющая, можно строить прогноз по следующей формуле [7]:

$$
\widetilde{Y}_{t+1}=Y_{t}+\Delta Y_{t}
$$

где

$$
\Delta Y_{t}=Y_{t}-Y_{t-1}
$$


Далее по мере накопления данных вместо последней разности ряда $\Delta Y_{t}$ можно вычислять усредненное изменение ряда за один период. По аналогии можно построить более сложные модели.

5. Простые средние. Для анализа временных рядов часто используются методики усреднения и сглаживания, призванные убрать различные флуктуации и шумы, мешающие анализу. Методы усреднения и, в частности, простые средние помогают делать прогноз, основываясь на усредненных значениях прошлых наблюдений [7].

Очевидно, что данные ряда можно сгладить различными способами. При этом неизвестными параметрами может быть, например, количество последних наблюдений, которые берутся для прогноза, или весовые коэффициенты, сопоставленные каждому из них. В общем случае для оценки количества параметров и их конкретных значений практически во всех методах идут путем подгонки модели к некоторым данным предыстории. Затем параметры проверяются и уточняются по мере поступления новых данных [3]. Говоря о различных параметрах, необходимо учитывать не только достигаемую при их использовании точность прогноза, но и степень сложности получившейся модели.

Ниже приведена формула построения прогноза с помощью нахождения среднего значения ряда:

$$
\widehat{Y}_{t+1}=\frac{1}{t} \sum_{i=0}^{t} Y_{i}
$$

Подобные прогнозы приемлемы в случае стационарного ряда, когда процессы, порождающие этот ряд, уже стабилизировались.

6. Скользящие средние. В отличие от метода простых средних, где усреднялись все известные члены ряда, в методе скользящих средних используется только некоторое количество самых последних наблюдений. Соответственно, при поступлении новых данных они включаются в усреднение, а такое же количество самых «старых» наблюдений исключается.

Формула прогноза на основе скользящего среднего порядка $\mathrm{n}$ имеет следующий вид [7]:

$$
\widehat{Y}_{t+1}=\frac{1}{n} \sum_{i=0}^{n-1} Y_{t-i}
$$

Иными словами, скользящее среднее порядка $\mathrm{n}$ есть среднее арифметическое последних $\mathrm{n}$ наблюдений. Следует отметить, что в подобных моделях неизбежно проявляется эффект «запаздывания», когда кривая скользящей средней не успевает реагировать на быстрые изменения направления ряда. Степень запаздывания зависит от периода усреднения - чем 
больше период, тем выше шансы запоздалой реакции на резкие движения. Таким образом, если в прогнозируемом ряде преобладают резкие движения, следует подбирать период скользящей средней как можно меньшим.

Если известно, что внутри интервала сглаживания имеется нелинейная тенденция, целесообразно применение взвешенных скользящих средних [3].

Главные достоинства данного метода - простота и наглядность.

7. Методы экспоненциального сглаживания. В целях дальнейшего улучшение точности прогноза в моделях, основанных на усреднении и сглаживании, целесообразно применение весовых коэффициентов, которые сопоставляются предшествующим членам временного ряда. Существуют также различные методы нахождения оптимальных весовых коэффициентов, которые позволяют добиваться улучшения прогнозов, адаптируясь к некоторым особенностям ряда. Во многих случаях используются последовательности весовых коэффициентов вида $w_{i}=\alpha^{i}$ где $\alpha<1$. Это позволяет добиться того, что наиболее свежие данные будут иметь наибольший вклад в формируемый прогноз. Для модели экспоненциально взвешенного скользящего среднего существуют методики регулировки скорости затухания $\alpha$. Так, в тех ситуациях, когда ошибка прогноза близка к нулю, скорость затухания $\alpha$ может быть увеличена, и наоборот [3].

Для уточнения прогноза обычно руководствуются принципом обратной связи, когда для корректировки используются ошибки в старых прогнозах. Таким образом, достигается постоянное обновление модели.

Формально процедуру экспоненциального сглаживания можно записать в следующем виде:

$$
\widehat{Y}_{t+1}=\alpha Y_{t}+(1-\alpha) \widehat{Y}_{t}
$$

или, переписав данное соотношение, получим

$$
\widehat{Y}_{t+1}=\widehat{Y}_{t}+\alpha\left(Y_{t}-\widehat{Y}_{t}\right)
$$

где

$\widehat{Y}_{t+1}-$ прогнозируемое значение на следующий период;

$\alpha$ - постоянная сглаживания $(0<\alpha<1)$;

$Y_{t}-$ наблюдение за текущий период $t$;

$\widehat{Y}_{t}-$ прежний прогноз на период $t$;

Таким образом, экспоненциально сглаживание есть старый прогноз, скорректированный на ошибку старого прогноза с учетом весового коэффициента [7]. Экспоненциальное 
сглаживание требует даже меньше арифметических операций, чем скользящие средние, а массив хранимой прошлой информации уменьшен до одного.

Также стоит отметить, что возможны различные стратегии выбора начального приближения. Так, можно, например, в качестве первого сглаживающего члена выбрать просто первое наблюдение или же взять некоторое усреднение первых наблюдений.

Главное достоинство модели прогнозирования, основанной на скользящей средней, заключается в том, что она способна адаптироваться к новому уровню процесса без значительных реакций на случайные отклонения. Однако данная модель дает значительную ошибку в случае, когда ряд имеет трендовую составляющую. Специально для этого случая существует несколько адаптивных моделей экспоненциального сглаживания. Например, двухпараметрическая модель Хольта [5]. В этом методе учитывается локальный линейный тренд, присутствующий во временных рядах. Идея метода состоит в том, что, если в данных присутствует локальный тренд, то, кроме оценки текущего уровня, необходимо оценивать также величину наклона. При этом постоянных сглаживания используется уже две. Это обеспечивает гибкость модели.

Прогноз на р периодов вперед, оценка уровня и тренда по модели Хольта описываются следующими выражениями, соответственно:

$$
\begin{gathered}
\hat{Y}_{t+p}=L_{t}+p T_{t} \\
L_{t}=\alpha Y_{t}+(1-\alpha)\left(L_{t-1}-T_{t-1}\right) \\
T_{t}=\beta\left(L_{t}-L_{t-1}\right)+(1-\beta) T_{t-1}
\end{gathered}
$$

где

$L_{t}-$ новая сглаженная величина;

$\alpha$ - постоянная сглаживания для данных $(0<\alpha<1)$;

$Y_{t}-$ наблюдение за текущий период $t$;

$\beta$ - постоянная сглаживания для оценки тренда $(0<\beta<1)$;

$T_{t}$ - оценка тренда;

$p$ - количество периодов вперед;

$\widehat{Y}_{t+p}-$ прогноз на $p$ периодов вперед;

Дальнейшее улучшение модели Хольта разработал в 1960 году Винтерс. Его подход заключался в том, чтобы учесть влияние сезонных колебаний. Естественно, бесплатных улучшений не бывает, за них приходится платить возрастающей сложностью модели. В данном случае для учета сезонных колебаний добавляется еще одно уравнение и, 
соответственно, еще один параметр - коэффициент сезонности. В результате мы получаем трехпараметрическую модель Винтерса [7]. Она задается следующей системой равенств:

$$
\begin{gathered}
L_{t}=\alpha \frac{Y_{t}}{S_{t-s}}+(1-\alpha)\left(L_{t-1}+T_{t-1}\right) \\
T_{t}=\beta\left(L_{t}-L_{t-1}\right)+(1-\beta) T_{t-1} \\
S_{t}=\gamma \frac{Y_{t}}{L_{t}}+(1-\gamma) S_{t-s} \\
\widehat{Y}_{t+p}=\left(L_{t}+p T_{t}\right) S_{t-s+p}
\end{gathered}
$$

где

$L_{t}-$ новая сглаженная величина;

$\alpha-$ постоянная сглаживания для данных;

$Y_{t}$ - наблюдение за текущий период $t$;

$\beta$ - постоянная сглаживания для оценки тренда;

$T_{t}$ - оценка тренда;

$\beta$ - постоянная сглаживания для оценки сезонности;

$S_{t}$ - оценка сезонности;

$s$ - длительность периода сезонного колебания;

$p$ - количество периодов вперед;

$\widehat{Y}_{t+p}-$ прогноз на $p$ периодов вперед;

Как видно из приведенных уравнений, данная модель является расширением модели Хольта. Величина $S_{t}$ призвана как раз учесть влияние сезонных колебаний и используется в последнем равенстве для корректировки прогноза. Что касается начальных значений, можно, например, взять первое значение сглаживания равным первому наблюдению, оценку тренда нулевой, а оценку сезонности единичной [7].

8. Авторегрессионные модели. Авторегрессионная модель порядка р имеет вид:

$$
Y_{t}=\phi_{0}+\phi_{1} Y_{t-1}+\phi_{2} Y_{t-2}+\cdots+\phi_{p} Y_{t-p}+\varepsilon_{t}
$$

где

$Y_{t}-$ значение временного ряда в момент времени t;

$\phi_{\tilde{i}}$ - оцениваемые коэффициенты;

$\varepsilon_{t}$ - ошибка, накапливающаяся от неучтенных переменных;

Свое название авторегрессионная модель получила ввиду того, что имеет вид регрессионной модели и использует в качестве независимой переменной запаздывающие 
значения зависимой переменной. Такие модели применимы для стационарных временных рядов, при этом коэффициент $\phi_{0}$ зависит от постоянного уровня ряда $\mu$ следующим образом:

$$
\phi_{0}=\mu\left(1-\phi_{1}-\phi_{2}-\cdots-\phi_{p}\right)
$$

Изначально порядок процесса авторегрессии, приемлемо описывающий наблюденный ряд, для нас может быть неизвестен. Для его определения используется анализ частной автокорреляционной функции, который основан на том, что хотя процесс авторегрессии имеет бесконечно протяженную функцию автокорреляции, тем не менее, он может быть описан при помощи $p$ ненулевых функций от автокорреляций. А именно, для процесса авторегрессии порядка $p$ частная автокорреляционная функция обращается в ноль при задержке, превышающей $p$ [2].

Автокорреляционные коэффициенты модели $\mathrm{AR}$ первого (a, б) и второго (в, г) порядка показаны на рис. 3. Можно заметить, что автокорреляционные коэффициенты модели AR имеют тенденцию затухать, стремясь к нулю, а частные автокорреляционные коэффициенты обращаются в ноль через промежуток времени, превышающий порядок модели [7]. В общем случае автокорреляционная функция стационарного процесса авторегрессии состоит из совокупности затухающих экспонент и затухающих синусоид [2].
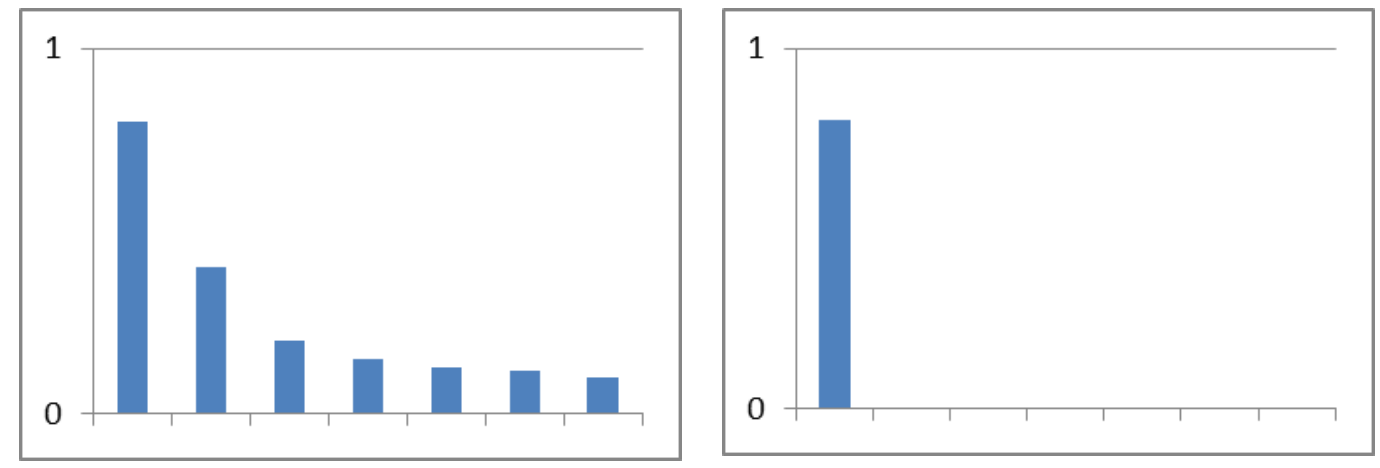

a)
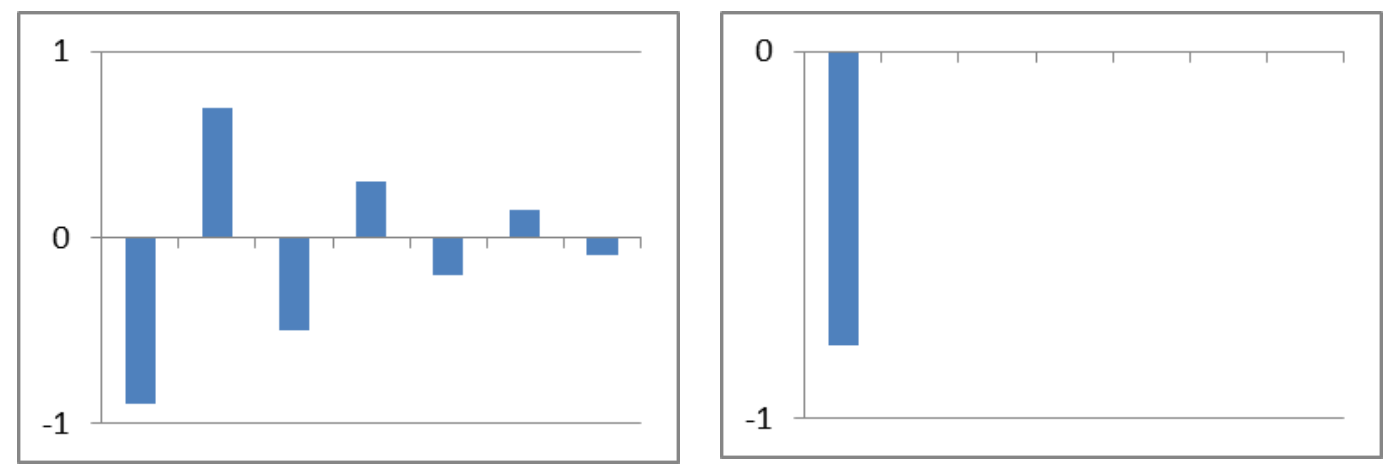

б) 

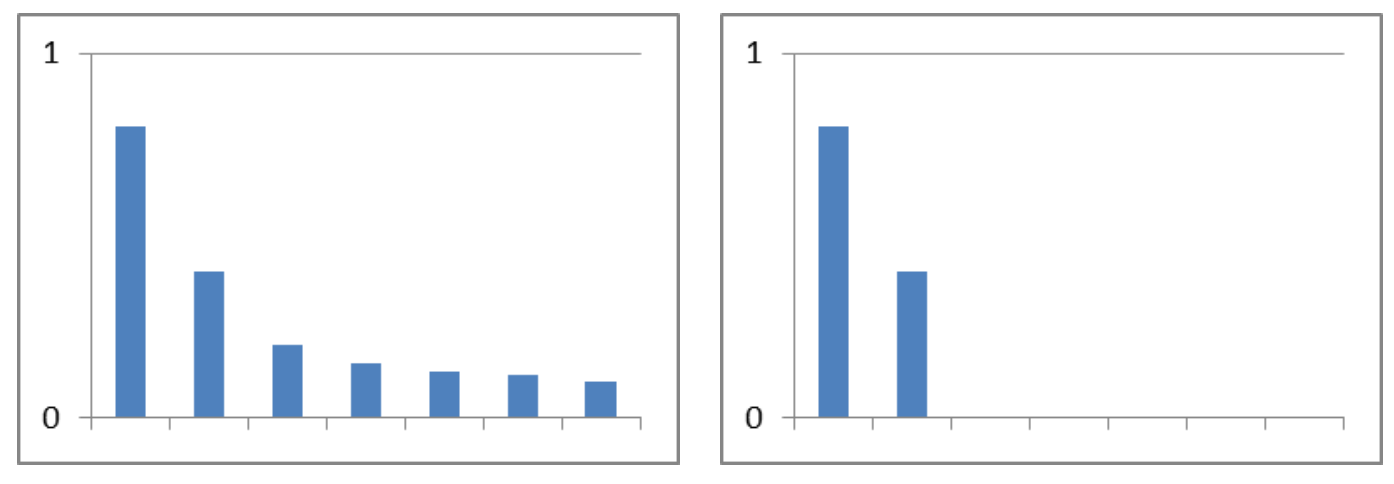

в)
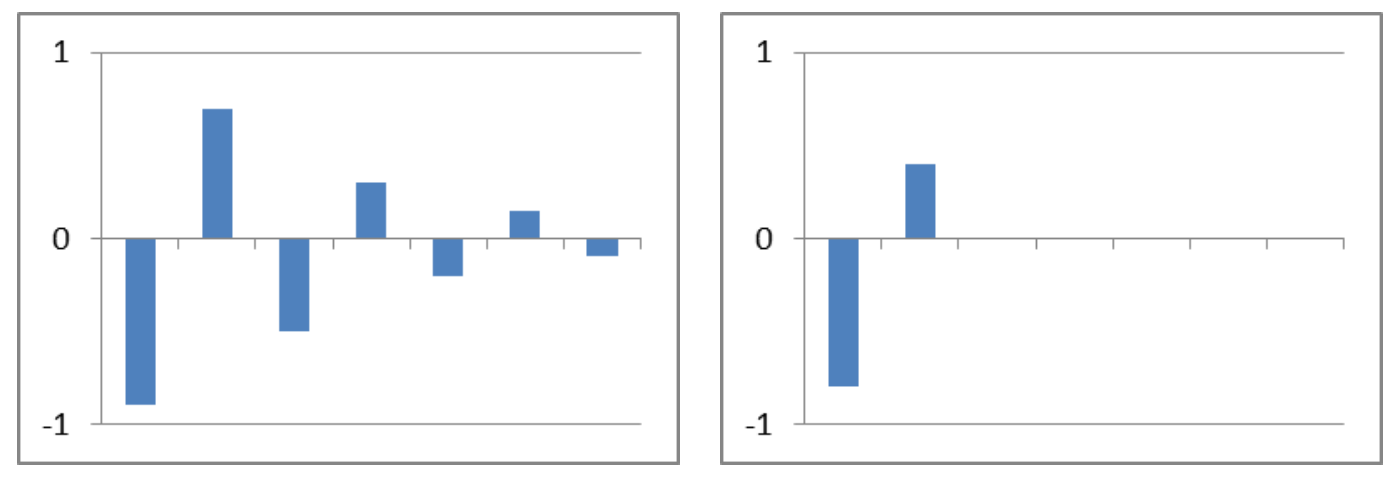

г)

Рис. 3. Коэффициенты автокорреляции и частной автокорреляции моделей авторегрессии первого и второго порядка

9. Модели со скользящим средним. Модель со скользящим средним порядка q имеет следующий вид:

$$
Y_{t}=\mu+\varepsilon_{t}-\omega_{1} \varepsilon_{t-1}-\omega_{2} \varepsilon_{t-2}-\cdots-\omega_{p} \varepsilon_{t-q}
$$

где

$Y_{t}-$ значение временного ряда в момент времени t;

$\mu$ - постоянное среднее ряда;

$\omega_{i}$ - оцениваемые коэффициенты;

$\varepsilon_{t}-$ ошибки в предыдущие моменты времени, которые в момент t включены в $Y_{t}$;

Данное уравнение отличается от уравнения авторегрессии тем, что $Y_{t}$ зависит от предыдущих значений ошибок, а не от значений отклика. Таким образом, модели со скользящим средним дают прогноз функции $Y_{t}$ на основе линейной комбинации прошлых ошибок, а не предыдущих значений самого ряда. Нужно также заметить, что в данном случае мы не налагаем каких-то строгих ограничений на коэффициенты $\omega_{i}-$ они не должны давать в сумме единицу, как и иметь положительный знак. 
Что касается термина «скользящее среднее», упомянутого в названии модели, это скорее исторически сложившееся название, не имеющее никакого отношения к процедуре вычисления скользящего среднего на основе усреднения всех или части последних наблюдений ряда [7].

Значение модели со скользящим средним заданного порядка удобно получать последовательным добавлением прошлых ошибок, которые были включены в прогноз прошлых наблюдений. На рис. 4 представлено теоретическое поведение коэффициентов автокорреляции и частичной автокорреляции модели со скользящим средним первого (a, б) и второго (в, г) порядка. Сравнив их с аналогичными графиками для модели авторегрессии, можно заметить, что в данном случае поведение коэффициентов совсем иное. Отличие в том, что у модели со скользящим средним коэффициенты автокорреляции обращаются в нуль сразу после первого (МА(1)) и второго $(\mathrm{MA}(2))$ периода, в то время как частные автокорреляции стремятся к нулю постепенно [1].

Мы видим, что коэффициенты автокорреляции для модели МА(q) равны нулю при запаздывании на период, превышающий порядок модели. Это важное свойство можно использовать при выборе порядка по экспериментальным данным.
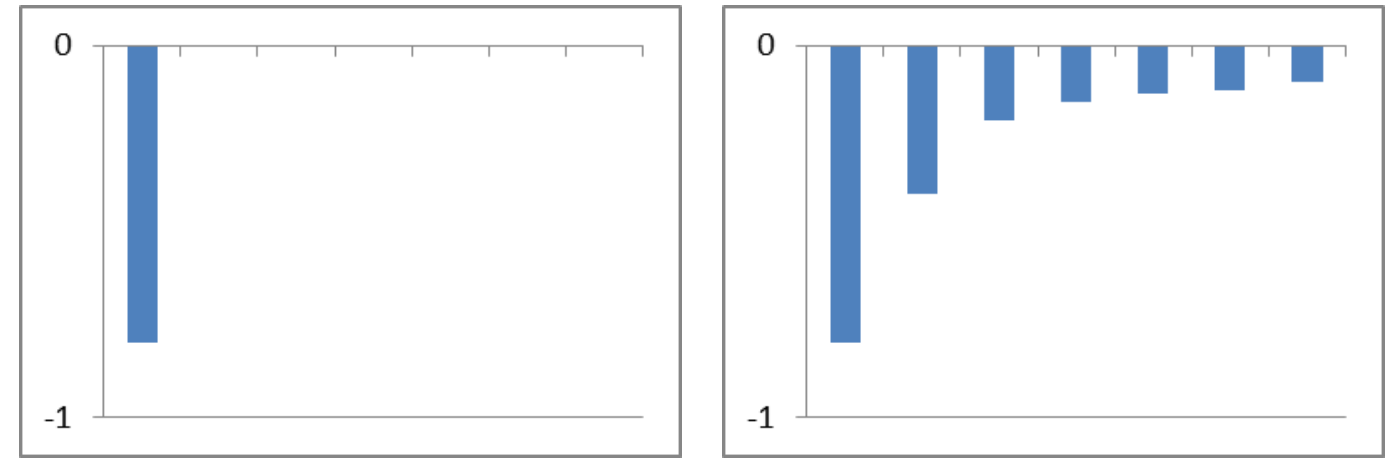

a)
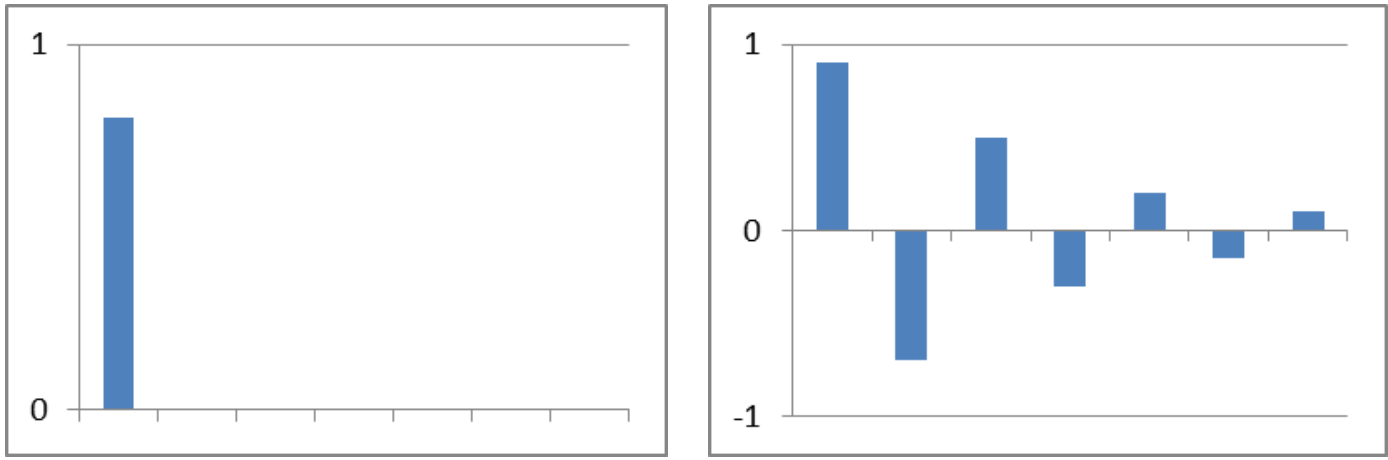

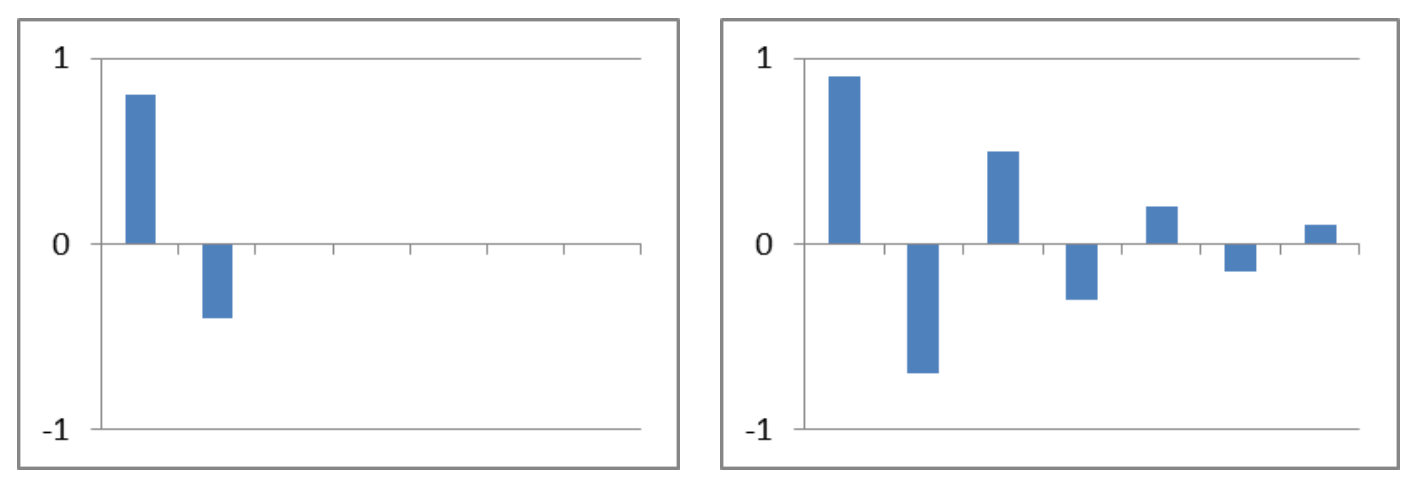

B)
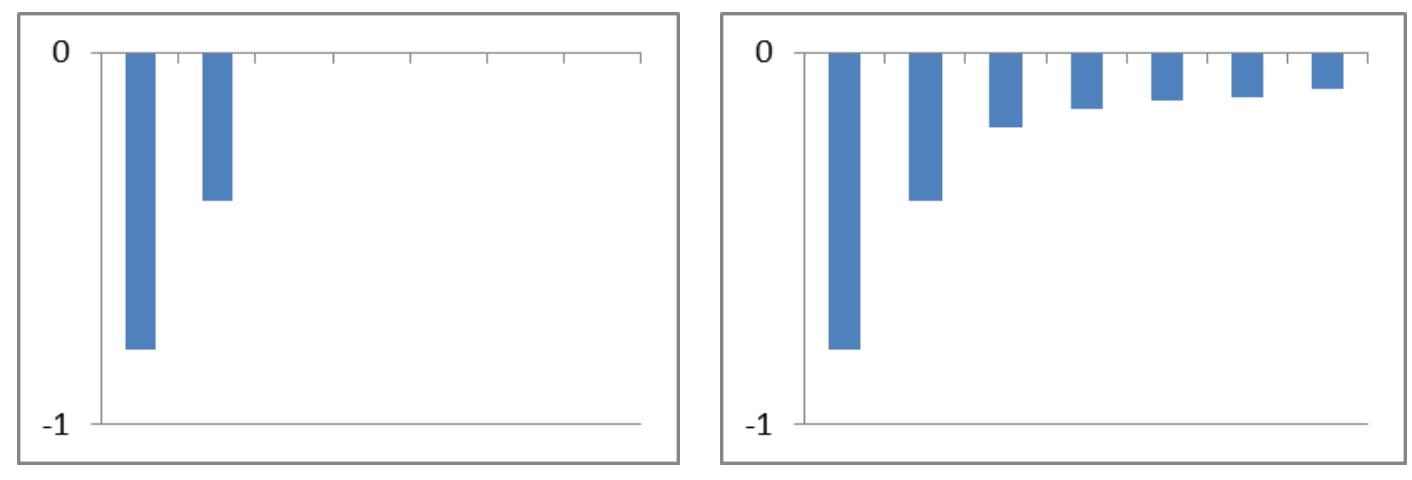

г)

Рис. 4. Коэффициенты автокорреляции и частной автокорреляции моделей скользящего среднего первого и второго порядка

10. Модель авторегрессии-скользящего среднего. Ввиду того, что в представлении временного ряда присутствует некий дуализм, а именно - один и тот же ряд может быть представлен двумя моделями, то сама собой напрашивается мысль «смешать» эти две модели, получив, таким образом, модель авторегрессии-скользящего-среднего. Если мы возьмем р членов в авторегрессионной части и q членов в части модели со скользящим средним, получившуюся смешанную модель обозначают ARMA(p, q). Модель имеет следующий вид [7]:

$$
Y_{t}=\phi_{0}+\phi_{1} Y_{t-1}+\phi_{2} Y_{t-2}+\cdots+\phi_{p} Y_{t-p}+\varepsilon_{t}-\omega_{1} \varepsilon_{t-1}-\omega_{2} \varepsilon_{t-2}-\cdots-\omega_{p} \varepsilon_{t-q}
$$

Таким образом, получившаяся модель зависит уже и от предыдущих значений отклика, и от предыдущих ошибок. Модель может быть использована для описания широкого спектра поведений стационарных временных рядов.

Что касается теоретических значений коэффициентов автокорреляции и частной автокорреляции, нетрудно догадаться, что для смешанной модели и те и другие будут плавно затухать, не обрываясь на каком-то шаге. 
Количество членов для каждой модели - AR члены и MA члены - определяется видом функции автокорреляции и частной автокорреляции конкретного ряда. Данный подход является частью методологии Бокса-Дженкинса по выбору наиболее подходящей модели [6]. Также в этом процессе необходимо учитывать так называемые критерии выбора модели. На практике результаты работы модели ухудшаются при попытке увеличить количество авторегрессионных членов или скользящего среднего, - это так называемая проблема «перепараметризации». Поэтому принято начинать подбор с наименьшего числа этих членов, при необходимости добавляя их постепенно. Соответственно, нужно стараться делать количество членов модели как можно меньшим [7].

11. Интегрированная модель авторегрессии-скользящего среднего. Многие эмпирические временные ряды (например, цены на бирже) ведут себя так, будто они не имеют фиксированного среднего. Но даже при этом в их поведении наблюдается некоторая однородность - любая часть временного ряда по своему поведению во многом подобна любой другой, если их привести к одному уровню. До этого момента мы говорили о построении модели стационарного ряда. На практике в первую очередь необходимо проверить ряд на стационарность, - т.е. изменяются ли его значения в окрестности некоторого неизменного уровня. Если мы делаем вывод о стационарности ряда, тогда мы можем использовать одну из разобранных моделей. В противном случае можно попытаться превратить нестационарный ряд в стационарный. Обычно это делается путем взятия разностей. В этом случае вместо исходного ряда $Y_{t}$ мы будем строить модель для ряда $\Delta Y_{t}$ :

$$
\Delta Y_{t}=Y_{t}-Y_{t-1}
$$

В некоторых случаях данную процедуру придется повторить некоторое количество раз обычно не больше двух-трех:

$$
\Delta^{2} Y_{t}=\Delta\left(\Delta Y_{t}\right)=Y_{t}-2 Y_{t-1}-Y_{t-2}
$$

Еще один распространенный прием - взятие логарифма разности.

Таким образом, если разность некоторого порядка есть стационарный смешанный процесс авторегрессии-скользящего среднего, мы получаем модель для нестационарных временных рядов - интегрированная модель авторегрессии-скользящего среднего [2]. Если обозначить количество применения оператора разности как $\mathrm{d}$, полученную модель можно обозначить стандартной записью $\operatorname{ARIMA}(\mathrm{p}, \mathrm{d}, \mathrm{q})$, где $\mathrm{p}$ - порядок авторегрессии, q - порядок членов скользящего среднего. Соответственно, при обнулении одного или двух параметров мы можем получать модели $\operatorname{ARMA}(\mathrm{p}, \mathrm{q}), \operatorname{AR}(\mathrm{p}), \mathrm{MA}(\mathrm{q})$. 
12. Критерии выбора модели. Как уже упоминалось ранее, процедура подбора модели ARMA основана на анализе коэффициентов автокорреляции и частной автокорреляции с теоретическими показателями, присущими моделям авторегрессии и скользящего среднего. Однако эта процедура неизбежно вносит некую долю субъективизма в выбор подходящей модели. К тому же нередки ситуации, когда две модели отвечают нужной структуре данных. В таком случае необходимо смотреть на среднеквадратичную ошибку модели, а также на количество на ее порядки.

Исходя из таких рассуждений, были разработаны несколько критериев, призванных помочь нам в процедуре подбора модели. Одним из таких критериев является информационный критерий Акаике (обозначается AIC), дающий числовую оценку пригодности модели (чем меньше числовое значение - тем лучше). Критерий имеет следующую формулу [6]:

$$
A I C=\ln \sigma^{2}+\frac{2}{n} r
$$

где

$\ln$ - натуральный логарифм;

$\mathrm{y}^{2}$ - остаточная сумма квадратов, деленая на количество наблюдений;

$n$ - количество наблюдений;

r - общее количество слагаемых в модели ARIMA

Другой распространенный критерий - Байесовский информационный критерий (обозначается ВIC) вычисляется похожим образом [7]:

$$
A I C=\ln \sigma^{2}+\frac{\ln n}{n} r
$$

Байесовский критерий более чувствителен к количеству параметров по сравнению с предыдущим критерием. Но на практике оба критерия зачастую дают похожие результаты.

13. Заключение. В статье рассмотрены несколько популярных классических моделей, предназначенных для анализа и прогнозирования временных рядов. Были рассмотрены модели усреднения и сглаживания, имеющие свои достоинства, такие как относительную вычислительную простоту и наглядность. Упомянуты сопутствующие подзадачи подбора и оптимизации параметров моделей, а также прилагающиеся к этим процедурам опасности, связанные с растущей сложностью модели прогнозирования. Мы видели, как из простых моделей авторегрессии и скользящего среднего, описывающих стационарные временные ряды, получается «смешанная» модель ARMA, которая с помощью преобразования временного ряда посредством разностного оператора распространялась на нестационарные 
временные ряды. Суть процедуры подбора подходящей модели сводилась к выбору порядка каждой составляющей путем анализа структуры функций автокорреляции и частной автокорреляции анализируемого ряда. Необходимо отметить, что как раз процедура изучения поведения коэффициентов корреляции и частной автокорреляции является зачастую трудно формализуемой и требует наличия определенных навыков. Рассмотренные критерии выбора модели, Акаике и Байесовский информационный критерии, не всегда способны помочь в выборе наилучшей модели. Хотя на практике при необходимости прогонки больших тестов, требующих полной автоматизации процесса, зачастую практикуется выбор наилучших моделей по информационному критерию.

\section{Список литературы}

1. Айвазян С.А., Мхитарян В.С. Прикладная статистика и основы эконометрики. М.: ЮНИТИ, 1998. 1000 с.

2. Бокс Дж., Дженкинс Г. Анализ временных рядов. Прогноз и управление. М.: Мир, 1974. T. 1,2. $601 \mathrm{c}$.

3. Грешилов А. А., Стакун В. А., Стакун А. А. Математические методы построения прогнозов. М.: Радио и связь, 1997. 112 с.

4. Елисеева И.И. Эконометрика, 2-е издание. М: Финансы и статистика, 2004. 344 с.

5. Лукашин Ю. П. Адаптивные методы краткосрочного прогнозирования временных рядов. М.: Финансы и статистика, 2003. 416 с.

6. Магнус Я.Р., Катышев П.К., Пересецкий А.А. Эконометрика. Начальный курс. М.: Дело, 2004. 576 с.

7. Ханк Д.Э., Уичерн Д.У., Райтс А.Дж. Бизнес-прогнозирование, 7-е издание. М.: Вильямс, 2003. 656 с.

8. Makridakis S., Wheelwright S.C., Hyndman R.J. Forecasting: Methods and Applications, 3rd edition. New York: Wiley, 1998. 656 p.

\section{UDK: 519.95}

Title: Some models of time series analysis and prediction

\section{Author(s):}

Igor V. Shevchenko (A.P. Ershov Institute of Informatics Systems)

Abstract: This paper presents some popular classical methods used for time series analysis and prediction. At first relatively simple models of averaging and smoothing are described, then autoregressive and moving average models, and a "mixed" autoregressive-moving-average model as a result of crossing of the latter two mentioned models. At last an autoregressive integrated moving average model is described. 
Keywords: forecasting, time series, averaging, exponential smoothing, autoregressive model, moving average 
